\title{
AN ELLIPTIC EQUATION WITH NO MONOTONICITY CONDITION ON THE NONLINEARITY
}

\author{
GREGORY S. SPRADLiN ${ }^{1}$
}

\begin{abstract}
An elliptic PDE is studied which is a perturbation of an autonomous equation. The existence of a nontrivial solution is proven via variational methods. The domain of the equation is unbounded, which imposes a lack of compactness on the variational problem. In addition, a popular monotonicity condition on the nonlinearity is not assumed. In an earlier paper with this assumption, a solution was obtained using a simple application of topological (Brouwer) degree. Here, a more subtle degree theory argument must be used.
\end{abstract}

Mathematics Subject Classification. 35J20, 35J60.

Received July 6, 2005.

\section{INTRODUCTION}

In this paper we consider an elliptic equation of the form

$$
-\Delta u+u=f(x, u), \quad x \in \mathbb{R}^{N},
$$

where $f$ is a "superlinear" function of $u$. For large $|x|$, the equation resembles an autonomous equation

$$
-\Delta u+u=f_{0}(u), \quad x \in \mathbb{R}^{N}
$$

Under weak assumptions on $f$ and $f_{0}$, we prove the existence of a nontrivial solution $u$ of (1.1) with $|u(x)| \rightarrow 0$ as $|x| \rightarrow \infty$.

Let $f$ satisfy

$\left(f_{1}\right) f \in C^{2}\left(\mathbb{R}^{N} \times \mathbb{R}, \mathbb{R}\right)$.

$\left(f_{2}\right) f(x, 0)=0=f_{q}(x, 0)$ for all $x \in \mathbb{R}^{N}$, where $f \equiv f(x, q)$.

$\left(f_{3}\right)$ If $N>2$, there exist $a_{1}, a_{2}>0, s \in(1,(N+2) /(N-2))$ with $\left|f_{q}(x, q)\right| \leq a_{1}+a_{2}|q|^{s-1}$ for all $q \in \mathbb{R}$, $x \in \mathbb{R}^{N}$. If $N=2$, there exist $a_{1}>0$ and a function $\varphi: \mathbb{R}^{+} \rightarrow \mathbb{R}$ with $\left|f_{q}(x, q)\right| \leq a_{1} \exp (\varphi(|q|))$ for all $q \in \mathbb{R}, x \in \mathbb{R}^{N}$ and $\varphi(t) / t^{2} \rightarrow 0$ as $t \rightarrow \infty$.

Keywords and phrases. Mountain-pass theorem, variational methods, Nehari manifold, Brouwer degree, concentration-compactness.

1 Department of Mathematics Embry-Riddle Aeronautical University Daytona Beach, Florida 32114-3900, USA; spradlig@erau.edu

(c) EDP Sciences, SMAI 2006 
$\left(f_{4}\right)$ There exists $\mu>2$ such that

$$
0<\mu F(x, q) \equiv \mu \int_{0}^{q} f(x, s) \mathrm{d} s \leq f(x, q) q
$$

for all $q \in \mathbb{R}, x \in \mathbb{R}^{N}$.

Let $f_{0} \in C^{2}(\mathbb{R}, \mathbb{R})$ with satisfy $\left(f_{1}\right)-\left(f_{4}\right)$ (except there is no dependence on $x$ ). Let $f$ also satisfy

$\left(f_{5}\right)\left(f(x, q)-f_{0}(q)\right) / f_{0}(q) \rightarrow 0$ as $|x| \rightarrow \infty$, uniformly in $q \in \mathbb{R}^{N} \backslash\{0\}$.

In order to state the theorem, we need to outline the variational framework of the problem. Define functionals $I_{0}, I \in C^{2}\left(W^{1,2}\left(\mathbb{R}^{N}, \mathbb{R}\right), \mathbb{R}\right)$ by

$$
\begin{aligned}
& I_{0}(u)=\frac{1}{2}\|u\|^{2}-\int_{\mathbb{R}^{N}} F_{0}(u(x)) \mathrm{d} x, \\
& I(u)=\frac{1}{2}\|u\|^{2}-\int_{\mathbb{R}^{N}} F(x, u(x)) \mathrm{d} x,
\end{aligned}
$$

where $\|u\|$ is the standard norm on $W^{1,2}\left(\mathbb{R}^{N}, \mathbb{R}\right)$ given by

$$
\|u\|^{2}=\int_{\mathbb{R}^{N}}|\nabla u(x)|^{2}+u(x)^{2} \mathrm{~d} x .
$$

Critical points of $I_{0}$ correspond exactly to solutions $u$ of (1.2) with $u(x) \rightarrow 0$ as $|x| \rightarrow \infty$, and critical points of $I$ correspond exactly to solutions $u$ of (1.1) with $u(x) \rightarrow 0$ as $|x| \rightarrow \infty$.

By $\left(f_{4}\right), F_{0}$ and $F$ are "superquadratic" functions of $q$, with, for example, $F(x, q) / q^{2} \rightarrow 0$ as $q \rightarrow 0$ and $F(x, q) / q^{2} \rightarrow \infty$ as $|q| \rightarrow \infty$ for all $x \in \mathbb{R}^{N}$, uniformly in $x$. Therefore $I(0)=I_{0}(0)=0$, and there exists $r_{0}>0$ with $I(u) \geq\|u\|^{2} / 3$ and $I_{0}(u) \geq\|u\|^{2} / 3$ for all $u \in W^{1,2}\left(\mathbb{R}^{N}\right)$ with $\|u\| \leq r_{0}$, and there also exist $u, u_{0} \in W^{1,2}\left(\mathbb{R}^{N}, \mathbb{R}\right)$ with $I_{0}\left(u_{0}\right)<0$ and $I(u)<0$. So the sets of "mountain-pass curves" for $I_{0}$ and $I$,

$$
\begin{gathered}
\Gamma_{0}=\left\{\gamma \in C\left([0,1], W^{1,2}\left(\mathbb{R}^{N}, \mathbb{R}\right)\right) \mid \gamma(0)=0, I_{0}(\gamma(1))<0\right\}, \\
\Gamma=\left\{\gamma \in C\left([0,1], W^{1,2}\left(\mathbb{R}^{N}, \mathbb{R}\right)\right) \mid \gamma(0)=0, I(\gamma(1))<0\right\},
\end{gathered}
$$

are nonempty, and the mountain-pass values

$$
\begin{aligned}
c_{0} & =\inf _{\gamma \in \Gamma_{0}} \max _{\theta \in[0,1]} I_{0}(\gamma(\theta)) \\
c & =\inf _{\gamma \in \Gamma} \max _{\theta \in[0,1]} I(\gamma(\theta))
\end{aligned}
$$

are positive.

We are now ready to state the theorem.

Theorem 1.1. If $f_{0}$ and $f$ satisfy $\left(f_{1}\right)-\left(f_{4}\right)$ and $f$ satisfies $\left(f_{5}\right)$, and if there exists $\alpha>0$ such that

$$
I_{0} \text { has no critical values in the interval }\left[c_{0}, c_{0}+\alpha\right)
$$

then there exists $\epsilon_{0}=\epsilon_{0}\left(f_{0}\right)>0$ with the following property: if $f$ satisfies

$$
\left|f(x, q)-f_{0}(q)\right|<\epsilon_{0}\left|f_{0}(q)\right|
$$

for all $x \in \mathbb{R}^{N}, q \in \mathbb{R}$, then (1.2) has a nontrivial solution $u \neq \equiv$ with $u(x) \rightarrow 0$ as $|x| \rightarrow \infty$.

As shown in [9], (1.12) holds in a wide variety of situations. 


\section{The missing monotonicity assumption}

One interesting aspect of Theorem 1.1 is a condition that is not assumed. We do not assume

$$
\begin{aligned}
& \text { For all } q \in \mathbb{R} \text { and } x \in \mathbb{R}^{N}, F_{0}(q) / q^{2} \text { is } \\
& \text { a nondecreasing function of } q \text { for } q>0 \text {; } \\
& F_{0}(q) / q^{2} \text { is a nonincreasing function of } q \text { for } q<0 \text {; } \\
& F(x, q) / q^{2} \text { is a nondecreasing function of } q \text { for } q>0 \text {; or } \\
& F(x, q) / q^{2} \text { is a nonincreasing function of } q \text { for } q<0 \text {. }
\end{aligned}
$$

This condition holds in the power case, $F_{0}(q)=|q|^{\alpha} / \alpha, \alpha>2$. The condition is due to Nehari.

If (1.13) were case, then for any $u \in W^{1,2}\left(\mathbb{R}^{N}, \mathbb{R}\right) \backslash\{0\}$, the mapping $s \mapsto I(s u)$ would begin at 0 at $s=0$, increase to a positive maximum, then decrease to $-\infty$ as $s \rightarrow \infty$. Defining

$$
\mathcal{S}=\left\{u \in W^{1,2}\left(\mathbb{R}^{N}, \mathbb{R}\right) \backslash\{0\} \mid I^{\prime}(u) u=0\right\},
$$

$\mathcal{S}$ would be a codimension-one submanifold of $E$, homeomorphic to the unit sphere in $W^{1,2}\left(\mathbb{R}^{N}, \mathbb{R}\right)$ via radial projection. $\mathcal{S}$ is known as the Nehari manifold in the literature. Any ray of the form $\{s u \mid s>0\}(u \neq 0)$ intersects $\mathcal{S}$ exactly once. All nonzero critical points of $I$ are on $\mathcal{S}$. Conversely, under suitable smoothness assumptions on $F$, any critical point of $I$ constrained to $\mathcal{S}$ would be a critical point of $I$ (in the large) (see [17]). Therefore, one could work with $\mathcal{S}$ instead of $W^{1,2}\left(\mathbb{R}^{N}, \mathbb{R}\right)$, and look for, say, a local minimum of $I$ constrained to $\mathcal{S}$ (which may be easier than looking for a saddle point of $I$ ). There is another way to use (1.13): for any $u \in \mathcal{S}$, the ray from 0 passing through $u$ can be used (after rescaling in $\theta$ ) as a mountain-pass curve along which the maximum value of $I$ is $I(u)$. Conversely, any mountain-pass curve $\gamma \in \Gamma$ intersects $\mathcal{S}$ at least once [6]. Therefore, one may work with points on $\mathcal{S}$ instead of paths in $\Gamma$. Since assumption (1.13) is so helpful, it is found in many papers, such as [1,5,20], and [18].

In the paper [17], a result similar to Theorem 1.1 was proven for the $N=1$ (ODE) case. The proof of Theorem 1.1 is similar except that a simple connectivity argument must be replaced by a degree theory argument [18]. proves a version of Theorem 1.1 under the assumption (1.13). Without 1.13, the manifold $\mathcal{S}$ must be replaced by a set with similar properties.

Define $B_{1}(0)=\left\{x \in \mathbb{R}^{N}|| x \mid<1\right\}$, and $\bar{\Omega}$ and $\partial \Omega$ to be, respectively, the topological closure and topological boundary of $\Omega$. It is a simple consequence of the Brouwer degree [7] that for any continuous function $h$ : $\overline{B_{1}(0)} \rightarrow \mathbb{R}^{N}$ with $h(x)=x$ for all $x \in \partial B_{1}(0)$, there exists $x \in B_{1}(0)$ with $h(x)=0$. We will need the following generalization:

Lemma 1.2. Let $h \in C\left(\overline{B_{1}(0)} \times[0,1], \mathbb{R}^{N}\right)$ with, for all $x \in \overline{B_{1}(0)}$ and $t \in[0,1]$,

(i) $h(x, 0)=x=h(x, 1)$.

(ii) $x \in \partial B_{1}(0) \Rightarrow h(x, t)=x$.

Then there exists a connected subset $C_{0} \subset \overline{B_{1}(0)} \times[0,1]$ with $(0,0),(0,1) \in C_{0}$ and $h(x, t)=0$ for all $(x, t) \in C_{0}$.

Using the Brouwer degree, it is clear that under the hypotheses of Lemma 1.2, for each "horizontal slice" $\overline{B_{1}(0)} \times\{t\}$ of the cylinder $\overline{B_{1}(0)} \times[0,1]$, there exists $x \in B_{1}(0)$ with $h(x, t)=0$. The conclusion of Lemma 1.2 does not follow from this observation. A generalization of Lemma 1.2 is known [16]: however, the reference may be difficult to find, so a proof is given here.

This paper is organized as follows: Section 2 contains the proof of Theorem 1.1. The proof of Lemma 1.2 is deferred until Section 3. 


\section{Proof of Theorem 1.1}

It is fairly easy to show that

$$
c \leq c_{0},
$$

where $c$ and $c_{0}$ are from (1.9)-(1.10): it is proven in [11] that there exists $\gamma_{1} \in \Gamma_{0}$ with $\max _{\theta \in[0,1]} I_{0}\left(\gamma_{1}(\theta)\right)=c_{0}$. Define the translation operator $\tau$ as follows: for a function $u$ on $\mathbb{R}^{N}$ and $a \in \mathbb{R}^{N}$, define let $\tau_{a} u$ be $u$ shifted by $a$, that is, $\left(\tau_{a} u\right)(x)=u(x-a)$. Let $\epsilon>0$. Let $\mathbf{e}_{1}=<1,0,0, \ldots, 0>\in \mathbb{R}^{N}$ and define $\tau_{R \mathbf{e}_{1}} \gamma_{1}$ by $\left(\tau_{R \mathbf{e}_{1}} \gamma_{1}\right)(\theta)=$ $\tau_{R_{\mathbf{e}_{1}}}\left(\gamma_{1}(\theta)\right)$. Then for large $R>0$, by $\left(f_{5}\right), \tau_{R_{\mathbf{e}_{1}}} \gamma_{1} \in \Gamma$ and $\max _{\theta \in[0,1]} I\left(\left(\tau_{R \mathbf{e}_{1}} \gamma_{1}\right)(\theta)\right)<c_{0}+\epsilon$. Since $\epsilon>0$ was arbitrary, $c \leq c_{0}$.

A Palais-Smale sequence for $I$ is a sequence $\left(u_{m}\right) \subset W^{1,2}\left(\mathbb{R}^{N}, \mathbb{R}\right)$ with $\left(I\left(u_{m}\right)\right)$ convergent and $\left\|I^{\prime}\left(u_{m}\right)\right\| \rightarrow 0$ as $m \rightarrow \infty$. It is well-known that $I$ fails the "Palais-Smale condition". That is, a Palais-Smale sequence need not converge. However, the following proposition states that a Palais-Smale sequence "splits" into the sum of a critical point of $I$ and translates of critical points of $I_{0}$ :

Proposition 2.1. If $\left(u_{m}\right) \subset W^{1,2}\left(\mathbb{R}^{N}, \mathbb{R}\right)$ with $I^{\prime}\left(u_{m}\right) \rightarrow 0$ and $I\left(u_{m}\right) \rightarrow a>0$, then there exist $k \geq 0$, $v_{0}, v_{1}, \ldots, v_{k} \in W^{1,2}\left(\mathbb{R}^{N}, \mathbb{R}\right)$, and sequences $\left(x_{m}^{i}\right)_{m \geq 1}^{1 \leq i \leq k} \subset \mathbb{R}^{N}$, such that

(i) $I^{\prime}\left(v_{0}\right)=0$;

(ii) $I_{0}^{\prime}\left(v_{i}\right)=0$ for all $i=1, \ldots, k$,

and along a subsequence (also denoted $\left(u_{m}\right)$ )

(iii) $\left\|u_{m}-\left(v_{0}+\sum_{i=1}^{k} \tau_{x_{m}^{i}} v_{i}\right)\right\| \rightarrow 0$ as $m \rightarrow \infty$;

(iv) $\left|x_{m}^{i}\right| \rightarrow \infty$ as $m \rightarrow \infty$ for $i=1, \ldots, k$;

(v) $\left|x_{m}^{i}-x_{m}^{j}\right| \rightarrow \infty$ as $m \rightarrow \infty$ for all $i \neq j$;

(vi) $I\left(v_{0}\right)+\sum_{i=1}^{k} I_{0}\left(v_{i}\right)=a$.

A proof for the case of $x$-periodic $F$ is found in [6], and essentially the same proof works here. Similar propositions for nonperiodic coefficient functions, for both ODE and PDE, are found in [1,5], and [19], for example. All are inspired by the "concentration-compactness" theorems of P.-L. Lions [12].

If $c<c_{0}$, then by standard deformation arguments [15], there exists a Palais-Smale sequence $\left(u_{m}\right)$ with $I\left(u_{m}\right) \rightarrow c$. By [11], the smallest nonzero critical value of $I_{0}$ is $c_{0}$. Applying Proposition 2.1, we obtain $k=0$, and $\left(u_{m}\right)$ has a convergent subsequence, proving Theorem 1.1. So assume from now on that

$$
c=c_{0}
$$

For $u \in L^{2}\left(\mathbb{R}^{N}, \mathbb{R}\right) \backslash\{0\}$ and $i \in\{1, \ldots, N\}$, define $\mathcal{L}_{i}$, the $i$ th component of the "location" of $u$, by

$$
\int_{\mathbb{R}^{N}} u^{2} \tan ^{-1}\left(x_{i}-\mathcal{L}_{i}(u)\right) \mathrm{d} x=0
$$

and the "location" of $u$ by

$$
\mathcal{L}(u)=\left(\mathcal{L}_{1}(u), \ldots, \mathcal{L}_{N}(u)\right) \in \mathbb{R}^{N} .
$$

The following lemma establishes the existence and continuity of $\mathcal{L}$.

Lemma 2.2. $\mathcal{L}$ is well-defined and continuous on $L^{2}\left(\mathbb{R}^{N}, \mathbb{R}\right) \backslash\{0\}$.

Proof. It suffices to show that $\mathcal{L}_{1}$ is well-defined and continuous on $L^{2}\left(\mathbb{R}^{N}, \mathbb{R}\right) \backslash\{0\}$. Let $u \in L^{2}\left(\mathbb{R}^{N}, \mathbb{R}\right) \backslash\{0\}$. By Leibniz's Theorem, the mapping $\phi: s \mapsto \int_{\mathbb{R}^{N}} u^{2} \tan ^{-1}\left(x_{1}-s\right) \mathrm{d} x$ is continuous, differentiable, and strictly decreasing, with

$$
\phi^{\prime}(s)=-\int_{\mathbb{R}^{N}} u^{2}(x) /\left(\left(x_{1}-s\right)^{2}+1\right) \mathrm{d} x<0
$$

$\phi(s) \rightarrow \mp \infty$ as $s \rightarrow \pm \infty$. Therefore $\mathcal{L}_{1}(u)$ is unique and well-defined. Let $\epsilon>0$ and $u_{m} \rightarrow u$. Now $\int_{\mathbb{R}^{N}} u^{2} \tan ^{-1}\left(x_{1}-\left(\mathcal{L}_{1}(u)+\epsilon\right)\right) \mathrm{d} x<0$. Since $u_{m}^{2} \rightarrow u^{2}$ in $L^{1}\left(\mathbb{R}^{N}, \mathbb{R}\right), \int_{\mathbb{R}^{N}} u_{m}^{2} \tan ^{-1}\left(x_{1}-(\mathcal{L}(u)+\epsilon)\right) \mathrm{d} x<0$ for 
large $m$, so for large $m, \mathcal{L}_{1}\left(u_{m}\right)<\mathcal{L}_{1}(u)+\epsilon$. Similarly, for large $m, \mathcal{L}_{1}\left(u_{m}\right)>\mathcal{L}_{1}(u)-\epsilon$. Since $\epsilon$ is arbitrary, $\mathcal{L}_{1}\left(u_{m}\right) \rightarrow \mathcal{L}_{1}(u)$

We are ready to begin the minimax argument. First we construct a mountain-pass curve $\gamma_{0}$ with some special properties:

Lemma 2.3. There exists $\gamma_{0} \in \Gamma_{0}$ such that for all $\theta \in[0,1]$,

(i) $I_{0}\left(\gamma_{0}(\theta)\right) \leq c_{0}$.

(ii) $\theta>0 \Rightarrow \gamma_{0}(\theta) \neq 0$.

(iii) $\theta \leq 1 / 2 \Rightarrow I_{0}(\gamma(\theta)) \leq c_{0} / 2$.

(iv) $\theta>0 \Rightarrow \mathcal{L}(\gamma(\theta))=0$.

Proof. By [10], there exists $\gamma_{1} \in \Gamma_{0}$ with $\max _{\theta \in[0,1]} I_{0}\left(\gamma_{1}(\theta)\right)=c_{0}$. Assume without loss of generality that $\gamma_{1}(\theta) \neq 0$ for $\theta>0$. By rescaling in $\theta$ if necessary, assume that $I_{0}\left(\gamma_{1}(\theta)\right) \leq c_{0} / 2$ for $\theta \leq 1 / 2$. Finally, define $\gamma_{0}$ by $\gamma_{0}(0)=0, \gamma_{0}(\theta)=\tau_{-\mathcal{L}\left(\gamma_{1}(\theta)\right)} \gamma_{1}(\theta)$ for $\theta>0$.

Assume $\epsilon_{0}$ in (1.12) is small enough so that for all $x \in \mathbb{R}^{N}$ and $\theta \in[0,1]$,

$$
I\left(\tau_{x}\left(\gamma_{0}(\theta)\right)<\min \left(2 c_{0}, c_{0}+\alpha\right) \text { and } I\left(\tau_{x}\left(\gamma_{0}(1)\right)\right)<0\right.
$$

where $\alpha$ is from (1.11).

\section{A substitute for $\mathcal{S}$}

Using the mountain-pass geometry of $I$ and the fact that Palais-Smale sequences of $I$ are bounded in norm [6], we construct a set which has similar properties to $\mathcal{S}$, described in Section 1. Let $\nabla I$ denote the gradient of $I$, that is, $(\nabla I(u), w)=I^{\prime}(u) w$ for all $u, w \in W^{1,2}\left(\mathbb{R}^{N}, \mathbb{R}\right)$. Here, $(\cdot, \cdot)$ is the usual inner product defined by $(u, w)=\int_{\mathbb{R}^{N}} \nabla u \cdot \nabla w+u w d x$. Let $\varphi: W^{1,2}\left(\mathbb{R}^{N}, \mathbb{R}\right) \rightarrow \mathbb{R}$ be locally Lipschitz, with $I(u) \geq-1 \Rightarrow \varphi(u)=1$ and $I(u) \leq-2 \Rightarrow \varphi(u)=0$. Let $\eta$ be the solution of the initial value problem

$$
\frac{\mathrm{d} \eta}{\mathrm{d} s}=-\varphi(\eta) \nabla I(u), \quad \eta(0, u)=u
$$

In [19] it is proven that $\eta$ is well-defined on $\mathbb{R}^{+} \times W^{1,2}\left(\mathbb{R}^{N}\right)$. Let $\mathcal{B}$ be the basin of attraction of 0 under the flow $\eta$, that is,

$$
\mathcal{B}=\left\{u \in W^{1,2}\left(\mathbb{R}^{N}, \mathbb{R}\right) \mid \eta(s, u) \rightarrow 0 \text { as } s \rightarrow \infty\right\}
$$

$\mathcal{B}$ is an open neighborhood of $0[19]$. Let $\partial \mathcal{B}$ be the topological boundary of $\mathcal{B}$ in $W^{1,2}\left(\mathbb{R}^{N}, \mathbb{R}\right)$. $\partial \mathcal{B}$ has some properties in common with $\mathcal{S}$. For example, for any $\gamma \in \Gamma, \gamma([0,1])$ intersects $\partial \mathcal{B}$ at least once.

A pseudo-gradient vector field for $I^{\prime}$ may be used in place of $\nabla I$, in which case $\mathcal{B}$ and $\partial \mathcal{B}$ would be different, but the ensuing arguments would be the same.

Let

$$
c^{+}=\inf \{I(u)|u \in \partial \mathcal{B},| \mathcal{L}(u) \mid \leq 1\} .
$$

The reason for the label " $c$ " will become apparent in a moment. From now on, let us assume

$$
I \text { has no critical values in }\left(0, c_{0}\right]=(0, c] \text {. }
$$

This will lead to the conclusion that $I$ has a critical value greater than $c_{0}$.

We claim that under assumptions (2.2) and (2.10),

$$
c^{+}>c_{0}
$$

We use arguments that are sketched here and found in more detail in [19] and [5]. 
To prove the claim, suppose first that $c^{+}<c_{0}$. Then there exists $u_{0} \in \partial \mathcal{B}$ with $I\left(u_{0}\right)<c_{0}$. By arguments in [19], there exists a large positive constant $P$ with

$$
I(u) \leq c_{0} \text { and }\|u\| \geq 2 P \Rightarrow I(\eta(s, u))<0 \text { for some } s>0, \text { and }\|\eta(s, u)\|>P
$$

for all $s>0$. Suppose $a>0$ and $\left\|I^{\prime}\left(\eta\left(s_{m}, U_{0}\right)\right)\right\| \geq a$ for some sequence $\left(s_{m}\right)$ with $s_{m} \rightarrow \infty$. Since $u_{0} \in \partial \mathcal{B}$, $\left\|\eta\left(u_{0}\right)\right\|<2 P$ for all $s>0$. $I^{\prime \prime}$ is bounded on bounded subsets of $W^{1,2}(\mathbb{R})$, so $I^{\prime}$ is Lipschitz on bounded subsets of $W^{1,2}(\mathbb{R})$. Therefore $I\left(\eta\left(s, u_{0}\right)\right)<0$ for some $s>0$. This is impossible since $u_{0} \in \partial \mathcal{B}$. Therefore $I^{\prime}\left(\eta\left(s, u_{0}\right) \rightarrow 0\right.$ as $s \rightarrow \infty$.

Define $u_{n}=\eta\left(n, u_{0}\right)$. Since $I^{\prime}\left(u_{n}\right) \rightarrow 0$ and $u_{n} \in \partial \mathcal{B}$, there exists $b \in\left(0, c_{0}\right)$ with $I\left(u_{n}\right) \rightarrow b$. By [11], $I_{0}$ has no critical values between 0 and $c_{0}$. Therefore, Proposition 2.1 , with $k=0$, implies that $\left(u_{n}\right)$ converges along a subsequence to a critical point $w$ of $I$ with $0<I(w)<c_{0}$. This contradicts assumption $(2.10)$.

Next, suppose that $c^{+}=c_{0}$. Then there exists a sequence $\left(u_{n}\right) \subset \partial \mathcal{B}$ with $\left|\mathcal{L}\left(u_{n}\right)\right| \leq 1$ for all $n$ and $I\left(u_{n}\right) \rightarrow c_{0}$ as $n \rightarrow \infty$. As above, $I^{\prime}\left(u_{n}\right) \rightarrow 0$ as $n \rightarrow \infty$; to prove, suppose otherwise. Then there exist $a>0$ and a subsequence of $\left(u_{n}\right)$ (also called $\left(u_{n}\right)$ ) along which $\left\|I^{\prime}\left(u_{n}\right)\right\|>a$. Since $\partial \mathcal{B}$ is forward- $\eta$-invariant [19], $\eta\left(1, u_{n}\right) \in \partial \mathcal{B}$ for all $n$. Since $\left(\eta\left(1, u_{n}\right)\right)_{n \geq 1}$ is bounded and $I^{\prime}$ is Lipschitz on bounded subsets of $W^{1,2}\left(\mathbb{R}^{N}, \mathbb{R}\right)$, for large $n, \eta\left(1, u_{n}\right) \in \partial \mathcal{B}$ with $I\left(\eta\left(1, u_{n}\right)\right)<c_{0}$. By the argument above, this implies that $I$ has a critical value in $\left(0, c_{0}\right)$, contradicting assumption $(2.2)$. Thus $I^{\prime}\left(u_{n}\right) \rightarrow 0$ as $n \rightarrow \infty$. Applying Proposition 2.1 and using the fact that $\left|\mathcal{L}\left(u_{n}\right)\right| \leq 1$ for all $n,\left(u_{n}\right)$ converges along a subsequence to a critical point of $I$, contradicting assumption (2.10). (2.11) is proven.

Let $R>0$ be big enough so that for all $x \in \partial B_{R}(0) \subset \mathbb{R}^{N}$ and $\theta \in[0,1]$,

$$
I\left(\tau_{x} \gamma_{0}(\theta)\right)<c^{+}
$$

This is possible by (1.12), (2.11), and Lemma 2.3(i). Define the minimax class

$$
\begin{gathered}
\mathcal{H}=\left\{h \in C\left(\overline{B_{R}(0)} \times[0,1], W^{1,2}\left(\mathbb{R}^{N}, \mathbb{R}\right)\right) \mid\right. \\
\text { for all } x \in \overline{B_{R}(0)} \text { and } t \in[0,1] \\
\quad t>0 \Rightarrow h(x, t) \neq 0 \\
\quad 0 \leq t \leq 1 / 2 \Rightarrow h(x, t)=\tau_{x} \gamma_{0}(t) \\
\quad x \in \partial B_{R}(0) \Rightarrow h(x, t)=\tau_{x} \gamma_{0}(t) \\
\\
\left.h(x, 1)=\tau_{x} \gamma_{0}(1)\right\}
\end{gathered}
$$

and the minimax value

We claim

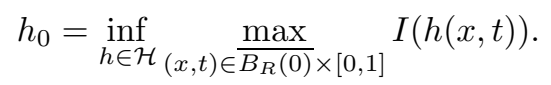

$$
c_{0}<c^{+} \leq h_{0}<\min \left(2 c_{0}, c_{0}+\alpha\right) .
$$

Proof of Claim. Define $\bar{h} \in \mathcal{H}$ by $\bar{h}(x, t)=\tau_{x}\left(\gamma_{0}(t)\right)$. Then $\bar{h} \in \mathcal{H}$ and by $(2.6), \max _{(x, t) \in \overline{B_{R}(0)} \times[0,1]} \bar{h}(x, t)<$ $\min \left(2 c_{0}, c_{0}+\alpha\right)$. Therefore $h_{0}<\min \left(2 c_{0}, c_{0}+\alpha\right)$.

Next, let $h \in \mathcal{H}$. By Lemma 1.2, and a suitable rescaling of $x$ and $t$, there exists a connected set $C_{2} \subset$ $B_{R}(0) \times[1 / 2,1]$ with $(0,1 / 2),(0,1) \in C_{2}$ and along which for all $(x, t) \in C_{2}$,

$$
\mathcal{L}(h(x, t))=0
$$

Joining $C_{2}$ with the segment $\{0\} \times[0,1 / 2]$, we obtain a connected set $C_{3} \subset B_{R}(0) \times[0,1]$ such that $(0,0),(0,1) \in$ $C_{3}$ and for all $(x, t) \in C_{3}, \mathcal{L}(h(x, t))=0 . C_{3}$ is not necessarily path-connected, so let $r>0$ be small enough so 
that for all

$$
\begin{gathered}
(x, t) \in N_{r}\left(C_{3}\right) \equiv\left\{(y, s) \in B_{R}(0) \times[0,1] \mid\right. \\
\left.\exists\left(x^{\prime}, t^{\prime}\right) \in B_{R}(0) \times[0,1] \text { with }\left|y-x^{\prime}\right|^{2}+\left(s-t^{\prime}\right)^{2}<r^{2}\right\} \\
|\mathcal{L}(h(x, t))|<1 .
\end{gathered}
$$

$N_{r}\left(C_{3}\right)$ is path-connected [21], so there exists a path $g \in C\left([0,1], N_{r}\left(C_{3}\right)\right)$ with $g(0)=(0,0), g(1)=(0,1)$, and $g(\theta) \in N_{r}\left(C_{3}\right)$ for all $\theta \in[0,1]$. If we define $\tilde{\gamma} \in \Gamma$ by $\tilde{\gamma}(\theta)=h(g(\theta))$, then $|\mathcal{L}(\tilde{\gamma}(\theta))|<1$ for all $\theta \in[0,1]$. Since $\tilde{\gamma}(0)=0$ and $I(\tilde{\gamma}(1))<0$, there exists $\theta^{*} \in[0,1]$ with $\tilde{\gamma}\left(\theta^{*}\right) \in \partial \mathcal{B}$. By the definition of $c^{+}(2.9), I\left(\tilde{\gamma}\left(\theta^{*}\right)\right) \geq c^{+}$.

Since $h$ was an arbitrary element of $\mathcal{H}, h_{0} \geq c^{+}$.

By standard deformation arguments, such as described in [15], there exists a Palais-Smale sequence $\left(u_{n}\right) \subset$ $W^{1,2}\left(\mathbb{R}^{N}, \mathbb{R}\right)$ with $I^{\prime}\left(u_{n}\right) \rightarrow 0$ and $I\left(u_{n}\right) \rightarrow h_{0}$ as $n \rightarrow \infty$. $c_{0}<h_{0}<\min \left(2 c_{0}, c_{0}+\alpha\right)$. Apply Proposition 2.1 to $\left(u_{n}\right)$. Since $I_{0}$ has no positive critical values smaller than $c_{0}[11], k \leq 1$. By $(2.10),\left(u_{n}\right)$ converges along a subsequence to a critical point $z$ of $I$, with $I(z)=h_{0}$. Theorem 1.1 is proven.

\section{A DEGREE-THEORETIC LEMMA}

Here, we prove Lemma 1.2. Let $h$ be as in the hypotheses of the lemma. For $l>0$, define $\mathcal{A}_{l} \subset \overline{B_{1}(0)} \times[0,1]$ by

$$
\mathcal{A}_{l}=\left\{(x, t) \in \overline{B_{1}(0)} \times[0,1]|| f(x, t) \mid<l\right\} .
$$

$\mathcal{A}_{l}$ is an open neighborhood of $(0,0)$. Let $C_{l}$ be the component of $\mathcal{A}_{l}$ containing $(0,0)$. We will prove the following claim:

$$
\text { For all } \epsilon>0,(0,1) \in C_{\epsilon} \text {. }
$$

Then we will use the $C_{\epsilon}$ 's to construct $C_{0}$. For $l>0$ and $t \in[0,1]$, define

$$
C_{l}^{t}=\left\{x \in \overline{B_{1}(0)} \mid(x, t) \in C_{l}\right\} .
$$

Fix $\epsilon \in(0,1)$. Define $\phi:[0,1] \rightarrow \mathbb{Z}$ by

$$
\phi(t)=d\left(h(\cdot, t), C_{\epsilon}^{t}, 0\right)
$$

where $d$ is the topological Brouwer degree [7]. We will prove $\phi(t)=1$ for all $t \in[0,1]$, in particular $\phi(1)=1$, so (3.2) is satisfied.

$f$ is continuous on a compact domain, so $f$ is uniformly continuous. Let $\rho>0$ be small enough so that for all $x \in \overline{B_{1}(0)}$ and $t_{1}, t_{2} \in[0,1]$,

$$
\left|t_{1}-t_{2}\right|<\rho \Rightarrow\left|h\left(x, t_{1}\right)-h\left(x, t_{2}\right)\right|<\epsilon / 4
$$

Clearly

$$
\phi(0)=d\left(i d, B_{\epsilon}(0), 0\right)=1 .
$$

Let $0 \leq t_{1}<t_{2} \leq 1$ with $t_{2}-t_{1}<\rho$. We will show $\phi\left(t_{1}\right)=\phi\left(t_{2}\right)$, proving that $\phi$ is constant, which by (3.6), implies (3.2).

$\Omega$ is nonempty. For all $x \in \partial C_{\epsilon}^{t_{1}},\left|h\left(x, t_{1}\right)\right|=\epsilon$, so by $(3.5)$,

$$
x \in \partial C_{\epsilon}^{t_{1}} \Rightarrow\left|h\left(x, t_{1}\right)\right| \geq \frac{3}{4} \epsilon .
$$

By the additivity property of $d[7]$,

$$
\begin{aligned}
\phi\left(t_{2}\right) & \equiv d\left(f\left(\cdot, t_{2}\right), C_{\epsilon}^{t_{2}}, 0\right) \\
& =d\left(f\left(\cdot, t_{2}\right), C_{\epsilon}^{t_{2}} \backslash \overline{C_{\epsilon}^{t_{1}}}, 0\right)+d\left(f\left(\cdot, t_{2}\right), C_{\epsilon}^{t_{1}} \cap C_{\epsilon}^{t_{2}}, 0\right) .
\end{aligned}
$$


We will show:

$$
\text { There does not exist } x \in C_{\epsilon}^{t_{2}} \backslash \overline{C_{\epsilon}^{t_{1}}} \text { with } h\left(x, t_{2}\right)=0 \text {. }
$$

Suppose such an $x$ exists. Then by $(3.5),|h|<\epsilon / 4$ on the segment $\{x\} \times\left[t_{1}, t_{2}\right] . x \in C_{\epsilon}^{t_{2}}$, so $\left(x, t_{2}\right) \in C_{\epsilon}$, and by the definition of $C_{\epsilon},\left(x, t_{1}\right) \in C_{\epsilon}$, and $x \in C_{\epsilon}^{t_{1}}$, contradicting $x \in C_{\epsilon}^{t_{2}} \backslash \overline{C_{\epsilon}^{t_{1}}}$. So (3.9) is true. Therefore by $(3.8)$,

$$
\phi\left(t_{2}\right)=d\left(f\left(\cdot, t_{2}\right), C_{\epsilon}^{t_{1}} \cap C_{\epsilon}^{t_{2}}, 0\right)
$$

By the same argument, switching the roles of $t_{1}$ and $t_{2}$,

$$
\phi\left(t_{1}\right)=d\left(f\left(\cdot, t_{1}\right), C_{\epsilon}^{t_{1}} \cap C_{\epsilon}^{t_{2}}, 0\right) .
$$

For all $t \in\left[t_{1}, t_{2}\right]$ and $x \in \partial C_{\epsilon}^{t_{1}} \cup \partial C_{\epsilon}^{t_{2}},(3.5)$ gives $\left|h\left(x, t_{1}\right)\right|>3 \epsilon / 4$ and $\left|h(x, t)-h\left(x, t_{1}\right)\right|<\epsilon / 4$. Therefore by the homotopy invariance property of the degree [7],

$$
\begin{aligned}
\phi\left(t_{1}\right) & =d\left(f\left(\cdot, t_{1}\right), C_{\epsilon}^{t_{1}} \cap C_{\epsilon}^{t_{2}}, 0\right) \\
& =d\left(f\left(\cdot, t_{2}\right), C_{\epsilon}^{t_{1}} \cap C_{\epsilon}^{t_{2}}, 0\right)=\phi\left(t_{2}\right) .
\end{aligned}
$$

$\phi(0)=1$ and $\phi\left(t_{1}\right)=\phi\left(t_{2}\right)$ for any $t_{1}<t_{2}$ with $t_{1}, t_{2} \in[0,1]$ and $t_{2}-t_{1}<\rho$. Therefore $\phi$ is constant, and $\phi(1)=1$. Therefore $(0,1) \in C_{\epsilon}$.

Now let

$$
C_{0}=\bigcap_{\epsilon>0} C_{\epsilon}
$$

Each $C_{\epsilon}$ is a connected set containing $(0,0)$ and $(0,1)$, so it is easy to show that $C_{0}$ is a connected set containing $(0,0)$ and $(0,1)$, and clearly for all $(x, t) \in C_{0}, h(x, t)=0$.

\section{REFERENCES}

[1] F. Alessio and P. Montecchiari, Multibump solutions for a class of Lagrangian systems slowly oscillating at infinity. Ann. Instit. Henri Poincaré 16 (1999) 107-135.

[2] A. Bahri and Y.-Y. Li, On a Min-Max Procedure for the Existence of a Positive Solution for a Certain Scalar Field Equation in $\mathbb{R}^{N}$. Revista Iberoamericana 6 (1990) $1-17$.

[3] P. Caldiroli, A New Proof of the Existence of Homoclinic Orbits for a Class of Autonomous Second Order Hamiltonian Systems in $\mathbb{R}^{N}$. Math. Nachr. 187 (1997) 19-27.

[4] P. Caldiroli and P. Montecchiari, Homoclinic orbits for second order Hamiltonian systems with potential changing sign. Comm. Appl. Nonlinear Anal. 1 (1994) 97-129.

[5] V. Coti Zelati, P. Montecchiari and M. Nolasco, Multibump solutions for a class of second order, almost periodic Hamiltonian systems. Nonlinear Ord. Differ. Equ. Appl. 4 (1997) 77-99.

[6] V. Coti Zelati and P. Rabinowitz, Homoclinic Orbits for Second Order Hamiltonian Systems Possessing Superquadratic Potentials. J. Amer. Math. Soc. 4 (1991) 693-627.

[7] K. Deimling, Nonlinear Functional Analysis. Springer-Verlag, New York (1985).

[8] M. Estaban and P.-L. Lions, Existence and non existence results for semilinear elliptic problems in unbounded domains. Proc. Roy. Soc. Edinburgh 93 (1982) 1-14.

[9] B. Franchi, E. Lanconelli and J. Serrin, Existence and Uniqueness of Nonnegative Solutions of Quasilinear Equations in $\mathbf{R}^{N}$. Adv. Math. 118 (1996) 177-243.

[10] L. Jeanjean and K. Tanaka, A Note on a Mountain Pass Characterization of Least Energy Solutions. Adv. Nonlinear Stud. 3 (2003) 445-455.

[11] L. Jeanjean and K. Tanaka, A remark on least energy solutions in $\mathbb{R}^{N}$. Proc. Amer. Math. Soc. 131 (2003) $2399-2408$.

[12] P.L. Lions, The concentration-compactness principle in the calculus of variations. The locally compact case. Ann. Instit. Henri Poincaré 1 (1984) 102-145 and 223-283.

[13] J. Mawhin and M. Willem, Critical Point Theory and Hamiltonian Systems. Springer-Verlag, New York (1989).

[14] P. Rabinowitz, Homoclinic Orbits for a class of Hamiltonian Systems. Proc. Roy. Soc. Edinburgh Sect. A 114 (1990) $33-38$.

[15] P. Rabinowitz, Minimax Methods in Critical Point Theory with Applications to Differential Equations, C.B.M.S. Regional Conf. Series in Math., No. 65, Amer. Math. Soc., Providence (1986).

[16] P. Rabinowitz, Théorie du degrée topologique et applications à des problèmes aux limites nonlineaires, University of Paris 6 Lecture notes, with notes by H. Berestycki (1975). 
[17] G. Spradlin, Existence of Solutions to a Hamiltonian System without Convexity Condition on the Nonlinearity. Electronic J. Differ. Equ. 2004 (2004) 1-13.

[18] G. Spradlin, A Perturbation of a Periodic Hamiltonian System. Nonlinear Anal. Theory Methods Appl. 38 (1999) $1003-1022$.

[19] G. Spradlin, Interacting Near-Solutions of a Hamiltonian System. Calc. Var. PDE 22 (2005) 447-464.

[20] E. Serra, M. Tarallo and S. Terracini, On the existence of homoclinic solutions to almost periodic second order systems. Ann. Instit. Henri Poincaré 13 (1996) 783-812.

[21] G. Whyburn, Topological Analysis. Princeton University Press (1964). 